\title{
VII. Lösungsansätze
}

\section{Rechtliche Begrenzungen}

Die steigenden Kassenkredite der Kommunen verdeutlichen, dass mittlerweile die historische Grundregel verletzt wird, keine Schulden zur Finanzierung laufender Ausgaben aufzunehmen. Die $1969 \mathrm{im}$ Grundgesetz verankerte „goldene Regel“ schrieb vor, dass sich der Staat nur für Investitionen verschulden dürfe ${ }^{1}$. Allerdings wurde der Investitionsbegriff stets weit ausgelegt, um den Handlungsspielraum zu erhöhen. Und wenn das nicht reichte, verkündete der Bundesfinanzminister eine Störung des gesamtwirtschaftlichen Gleichgewichts. Damit sollte eine antizyklische Finanzpolitik ermöglicht werden. In der Praxis führte es allerdings dazu, dass Bund und Länder ihre Kredite auch für laufende Ausgaben langfristig aufnahmen. Kassenkredite früher hätte man gesagt: „schwebende Schuld“ - existierten dadurch auf Bundes- oder Landesebene mit Ausnahme der zu vernachlässigenden Betriebsmittelkredite nicht.

Bis zur Haushaltsreform von 1969 gab es bei Bund und Ländern einen ordentlichen und außerordentlichen Haushalt. Im letzteren wurden - ähnlich $\mathrm{zu}$ den kommunalen Vermögenshaushalten - die Investitionen veranschlagt, die der Staat mit fundierten Krediten, also klassischerweise mit Anleihen, finanzierte. Kredite sollten nur für „werbende“ oder „produktive“ Investitionen aufgenommen werden. Fritz Schäffer meinte daher, Deficit spending sei „durch das Grundgesetz in der deutschen Finanzpolitik untersagt ${ }^{\text {“2 }}$. Allzu oft wurde aber bereits damals auf die Umwegrentabilität verwiesen. Arbeitsplatzeffekte und höhere Steuereinnahmen mussten herhalten, um eine Rentierlichkeit zu belegen. Der Nachweis konnte selbstredend nie erbracht werden. Seitdem es die Unterscheidung in ordentlichen und außerordentlichen Haushalt nicht mehr gab, konnten die Haushaltslücken mit einer fundierten Schuldaufnahme geschlossen werden. Damit verschleierte insbesondere der Bund seine problematische Finanzlage.

${ }^{1}$ Vgl. hierzu und zum Folgenden Elmar Dönnebrink u.a., Entstehungsgeschichte und Entwicklung des BMF-Konzepts, in: Christian Kastrop/Gisela Meister-Scheufelen/ Margaretha Sudhof (Hrsg.), Die neuen Schuldenregeln im Grundgesetz. Zur Fortentwicklung der bundesstaatlichen Finanzbeziehungen, Berlin 2010, S. 22-61, hier S. 23-30.

${ }^{2}$ Rede zur Einbringung des Haushalts von Fritz Schäffer am 9.11.1950, in: Haushaltsreden. Die Ära Schäffer, 1949 bis 1957, bearb. von Kurt-Dieter Wagner u.a., Bonn 1992, S. 81. 
Nicht nur die „goldene Regel“ des Grundgesetzes, die Verschuldung auf die Höhe der Investitionen zu begrenzen, hat sich als unwirksam erwiesen ${ }^{3}$, sondern auch die Maastricht-Kriterien waren keine wirksame Schuldenbremse. Zwar haben sie sowohl in Deutschland als auch in anderen europäischen Staaten in der zweiten Hälfte der 1990er Jahre eine konsolidierende Finanzpolitik gefördert, doch mit der Aufnahme in den Kreis der EuroLänder wurden die Zügel häufig wieder gelockert. Über die Begrenzung der Schulden auf maximal 60 Prozent des Bruttoinlandsprodukts redet heute niemand mehr. Nur die drei Prozent-Regel für das jährliche Defizit wird von der Europäischen Kommission noch hochgehalten, von den meisten Mitgliedstaaten aber spätestens seit der 2008 einsetzenden Wirtschaftskrise ignoriert. Dabei haben Deutschland und Frankreich eine unrühmliche Rolle gespielt, als sie im Jahr 2005 eine erhebliche Lockerung der MaastrichtKriterien durchsetzten".

Nach langer Diskussion hat die zweite Große Koalition im Sommer 2009 die sogenannte Schuldenbremse verabschiedet ${ }^{5}$. Demnach darf die Nettoneuverschuldung des Bundes ab 2016 nur noch 0,35 Prozent des Bruttoinlandsprodukts betragen, während die Bundesländer sich ab 2020 überhaupt nicht mehr verschulden dürfen ${ }^{6}$. Diese Klauseln sind vorbereitet worden, als die Steuereinnahmen sprudelten und der Bund und die Mehrzahl der Bundesländer entweder bereits über einen ausgeglichenen Haushalt verfügten oder kurz davor standen. Dieser positive Zustand der öffentlichen Finanzen und der Umstand, dass die Regel erst in einigen Jahren greifen wird, haben die Zustimmung zur Schuldenbremse ungemein erleichtert. Die 2009/10 stark angestiegene Verschuldung lässt Zweifel aufkommen, ob die Zielvorgaben der Schuldenbremse zu erreichen sind. Mit einiger Berechtigung ist $\mathrm{zu}$ fragen, warum ausgerechnet dieses Mal die geltenden Regeln eingehalten werden sollten, da die „Erfahrung der letzten 40 Jahre“ zeigt, dass "gesetzlich normierte Schuldengrenzen die Neuverschuldung“ mitnichten verhindern ${ }^{7}$.

${ }^{3}$ Vgl. Kai A. Konrad, Überlegungen zur „Goldenen Regel“ zur Schuldenbegrenzung, in: ders./Jochimsen (Hrsg.), Föderalismuskommission II, S. 77-89.

${ }^{4}$ Vgl. Bajohr, Grundriss Staatliche Finanzpolitik, S. 51.

${ }^{5}$ Vgl. den Überblick bei Kastrop/Meister-Scheufelen/Sudhof (Hrsg.), Schuldenregeln.

${ }^{6}$ Vgl. Monatsbericht des Bundesministeriums der Finanzen vom März 2009, S. 36-44, hier S. 39 .

${ }^{7}$ Stefan Korioth, Die neuen Schuldenbegrenzungsregeln für Bund und Länder symbolische Verfassungsänderung oder gelungene Schuldenbremse?, in: Perspektiven der Wirtschaftspolitik 11 (2010), S. 270-287, hier S.285. Vgl. dagegen optimistischer Mark Hallerberg, The German Debt Brake in Comparative Perspective. When Do Fiscal 


\section{Insolvenz}

Man könnte den Schuldenstaat freilich auch durch die Einführung eines förmlichen Insolvenzverfahrens für Gebietskörperschaften überwinden. Dieser Ansatz wird immer dann diskutiert, wenn ein größeres Land in finanzpolitische Turbulenzen gerät wie zum Beispiel Argentinien 2002 oder Griechenland 2010. Trotz mehrerer Anläufe, die vom Internationalen Währungsfonds ausgingen, ist die Staatsinsolvenz bisher nicht eingeführt worden ${ }^{8}$. Für die Mitgliedstaaten der Europäischen Union (EU) fordert insbesondere die Wissenschaft ein geordnetes Insolvenzverfahren". So bezeichnet das „Plenum der Ökonomen“ in seiner Stellungnahme zur EU-Schuldenkrise "die Möglichkeit einer Staatsinsolvenz mit anschließender Umschuldung“"

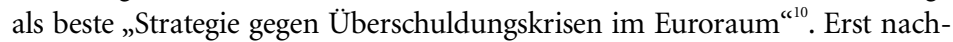
dem „private Gläubiger zumindest auf einen Teil ihrer Forderungen “verzichtet haben, „sind Hilfskredite der EU geboten“. Obwohl Kanzlerin Merkel und Finanzminister Schäuble genau dies anmahnten und das im Maastrichter Vertrag verankerte No-Bail-out-Prinzip nicht aufgeben wollten, bewegt sich die EU mit dem jüngst beschlossenen Europäischen Stabilisierungsmechanismus (ESM) ,in Richtung einer zentralisierten Wirtschafts- und Finanzpolitik bei vergemeinschafteter Haftung “'11. Wahrscheinlich wird es auch in Zukunft nicht zu einem europäischen oder internationalen Insolvenzrecht für Staaten kommen, da keine Regierung bereit sein dürfte, Entscheidungsbefugnisse des eigenen Landes aufzugeben.

In föderal aufgebauten Staaten besteht die Möglichkeit, dass die Einzelstaaten beziehungsweise Länder und die Kommunen insolvenzfähig sind. In den USA waren die Bundesstaaten früher häufig bankrott ${ }^{12}$. Anfang 2011

Rules Suceed?, in: Kastrop/Meister-Scheufelen/Sudhof (Hrsg.), Schuldenregeln, S. 287303, hier S. 300.

${ }^{8}$ Vgl. Alexander Szodruch, Staateninsolvenz und private Gläubiger: Rechtsprobleme des Private Sector Involvement bei staatlichen Finanzkrisen im 21. Jahrhundert, Berlin 2008.

${ }^{9}$ Vgl. z.B. Wissenschaftlicher Beirat beim Bundesministerium für Wirtschaft und Technologie, Gutachten 01/11: Überschuldung und Staatsinsolvenz in der Europäischen Union, Berlin 2011.

${ }^{10}$ Hier und nachfolgend: www.wiso.uni-hamburg.de/lucke/?p=581.

${ }^{11}$ Gemeinschaftsdiagnose vom Frühjahr 2011: Aufschwung setzt sich fort. Europäische Schuldenkrise noch ungelöst, Halle 2011, S.51. Die Formulierungen bezüglich der Collective Action Clauses sind im ESM so weich gefasst, dass private Gläubiger auch ab 2013 wohl kaum auf Forderungen verzichten müssen. Sie haben im Grunde nur eine gesichtswahrende Funktion für die deutsche Bundesregierung.

${ }^{12}$ Vgl. Manes, Staatsbankrotte, S. 16, und Wright, Nation under debt, S. $275 \mathrm{ff}$. 
wurde von den Republikanern ein Gesetzentwurf zur Insolvenzmöglichkeit der Bundesstaaten angekündigt ${ }^{13}$. Amerikanische Kommunen sind bereits insolvenzfähig und melden tatsächlich auch Insolvenz $\mathrm{an}^{14}$. Auch in Deutschland wird dieser Ansatz seit einiger Zeit diskutiert. Während eine Reihe von Ökonomen und Juristen ${ }^{15}$ die positiven Effekte der Insolvenzfähigkeit wie zum Beispiel die Beteiligung der Gläubiger an der Sanierung und die disziplinierende Wirkung auf das Ausgabeverhalten betonen, lehnt insbesondere der Städtetag diesen Ansatz entschieden $a b^{16}$, da sich die Kreditaufnahme schlagartig verteuern würde und die kommunale Selbstverwaltung grundsätzlich in Frage gestellt wäre.

In der Tat könnte eine Insolvenz die Selbstverwaltung der betroffenen Kommune temporär außer Kraft setzen. Zwar drohte aufgrund der „Systemrelevanz" keine Abwicklung der Kommune, sondern es ginge - wie in den USA - lediglich um die Sanierung des Haushalts. Allerdings würde das für die betroffene Gemeinde zuständige Bundesland wohl einen Staatskommissar einsetzten, der die Geschäfte sowohl vom Oberbürgermeister als auch vom Stadtrat übernähme.

Selbstverständlich können die Aufgaben einer Kommune nicht einfach abgewickelt werden. Das ist ein zentraler Unterschied zur überwiegenden Mehrheit der privaten Unternehmen. Die Produkte und Dienstleistungen eines Automobilunternehmens oder einer Kaufhauskette können relativ leicht durch die Konkurrenz ersetzt werden, gerade weil es auf diesen Märkten heftigen Wettbewerb und eventuell sogar Überproduktion gibt. Hingegen gelten große Banken als systemrelevant, weswegen sie in der Finanzkrise

${ }^{13}$ Vgl. Agentur Reuters vom 22.1.2011, 11.43 Uhr.

${ }^{14}$ Beispiele sind Vallejo und Harrisburg. Vgl. Die Zeit vom 19.8.2010 und Handelsblatt vom 25.6.2010.

${ }^{15}$ Vgl. Christian Magin, Kommunale Rechnungslegung. Konzeptionelle Überlegungen, Bilanzanalyse, Rating und Insolvenz, Wiesbaden 2011, S. 202-231; Hannes Rehm/Sigrid Matern-Rehm, Kommunalfinanzen, Wiesbaden 2010, S.175-187; Stefan Niederste Frielinghaus, Die kommunale Insolvenz als Sanierungsansatz für die öffentlichen Finanzen, Stuttgart 2007; Blankart, Föderalismus, S. 169-178; Jens Lehmann, Die Konkursfähigkeit juristischer Personen des öffentlichen Rechts, Berlin 1999, insbesondere S.91-106. Blankart und Lehmann erläutern aktuelle und historische Beispiele von Kommunalinsolvenzen, vor allem Leukerbad in der Schweiz im Jahr 1998 und Glashütte zu Beginn der Weltwirtschaftskrise 1929.

${ }^{16} \mathrm{Vgl}$. Arbeitsgemeinschaft der kommunalen Spitzenverbände NRW, Stellungnahme zur Sachverständigenanhörung am 5.11.2008 im Ausschuss für Kommunalpolitik und Verwaltungsstrukturreform des Landtages Nordrhein-Westfalen „Rechtliche Zuverlässigkeit einer Überschuldung einzelner Kommunen gem. \$75 Abs. 7 GO NRW“, Köln 2008. 
von 2008/09 staatliche Hilfen erhalten haben. Eine Kommune ist ebenfalls systemrelevant, zumindest für ihre Bürger. Die meisten kommunalen Aufgaben können nicht einfach eingestellt werden. So müssen beispielsweise Personalausweise weiterhin ausgegeben werden, und der Müll würde bei Nichtabholung spätestens nach einer Woche anfangen zu stinken.

Die Frage stellt sich, wann eine Kommune Insolvenz anmelden müsste. Grundsätzlich gibt es zwei mögliche Ursachen: Liquiditätsschwierigkeiten oder bilanzielle Überschuldung. Bei einer kommunalen Insolvenzfähigkeit wäre es nicht unwahrscheinlich, dass insbesondere Städte mit hohen strukturellen Haushaltsdefiziten von den Banken keine Kassenkredite mehr bekämen. Dann würde der Insolvenzfall sofort eintreten, da die Stadt ihren Verpflichtungen nicht mehr nachkommen könnte. Die bilanzielle Überschuldung als Insolvenzmaßstab gestaltet sich bei einer Kommune deutlich schwieriger. Viele Städte haben ihr Vermögen im Rahmen der Umstellung auf das kaufmännische Rechnungswesen mittlerweile bewertet ${ }^{17}$. Das kommunale Eigenkapital ist anders anzusehen als das Eigenkapital eines Unternehmens, welches tatsächlich eine wichtige Funktion für den Gläubigerschutz hat ${ }^{18}$. Ein Großteil des kommunalen Vermögens ist nicht rentabel und faktisch unverkäuflich. Zwar lässt sich allein ein Museum mühelos auf hunderte von Millionen Euro bewerten, realisierbar sind diese Werte aber in der Regel nicht. So wollte die Stadt Krefeld ein Gemälde zur Sanierung des Kunstmuseums verkaufen ${ }^{19}$. Der Schätzwert für „Das Parlamentsgebäude in London" von Claude Monet lag bei 20 Millionen Euro. Der Aufschrei war jedoch gewaltig. Der Deutsche Museumsbund sah in diesem Vorgang einen Verstoß gegen den internationalen Museums-Ehrenkodex. Dieser Kritik schloss sich Kulturstaatsminister Bernd Neumann (CDU) an. Der Kulturausschuss des Landtags äußerte sein „Befremden“ über die Pläne. Schließlich sprach sich der Krefelder Stadtrat einstimmig gegen die Veräußerung des Kunstwerks aus. Da also ein Großteils des kommunalen Vermögens nicht veräußert werden kann, stehen scheinbar reiche Städte nicht unbedingt besser da als Städte mit einem negativen Eigenkapital.

${ }^{17}$ Vgl. Philipp Häfner, Doppelte Buchführung für Kommunen, München ${ }^{4} 2009$, S. $22 \mathrm{f}$.

${ }^{18}$ Vgl. Oliver Kiamann/Stefan Wielenberg, Sind die Regeln der externen Unternehmensrechnung auf die kommunale Rechnungslegung übertragbar?, in: Zeitschrift für Betriebswirtschaft 80 (2010), S.237-261, hier S.254f., sowie Christian Magin, Kommunale Doppik: (Miss-)Verständnisse und Weiterentwicklungen, in: Der Gemeindehaushalt 108 (2007), S. 175-180, hier S. 177f.

${ }^{19}$ Vgl. hierzu und zum Folgenden Spiegel online vom 3.11.2006 sowie Der Spiegel vom 16.10.2006, S. 19 . 
Was würde die Insolvenz einer deutschen Kommune bedeuten? Nachdem die Stadt aufgrund erheblicher Liquiditätsschwierigkeiten Insolvenz angemeldet hat, setzt das Land einen Staatskommissar ein, der seinerseits einen erfahrenen Insolvenzverwalter mit der Erarbeitung eines Sanierungsplans beauftragt. Da ein Viertel der Ausgaben der Kommune durch das Personal verursacht wird, setzt der Insolvenzverwalter als erstes hier an und lädt die Beschäftigten zu einer Personalversammlung ein. Dort werden eine Gehaltskürzung von 20 Prozent für alle Mitarbeiter, 2500 betriebsbedingte Kündigungen sowie der Verkauf nicht betriebsnotwendiger Teile des Konzerns Stadt angekündigt. Der Insolvenzverwalter leitet eine großflächige Privatisierung ein: Krankenhäuser, Pflegeheime, Stadtwerke, Flughafen, Messe, Wohnungsunternehmen, Parkhäuser, Hafen, Stadtentwässerung und Müllabfuhr werden in einem Bieterverfahren ausgeschrieben. Der Verkaufserlös wird eingesetzt, um die Stadt komplett zu entschulden, und zwar sowohl von den kurz- als auch von den langfristigen Krediten. Darüber hinaus soll die Eigenkapitalausstattung der Kommune deutlich erhöht werden, um sie für die Zukunft krisensicherer zu machen. Auch die Gläubiger tragen zur Sanierung bei. Sie müssen auf einen nicht geringen Teil ihrer Forderungen verzichten, was üblicherweise eine wichtige Intention von Unternehmensinsolvenzen ist.

Das Sanierungsprogramm sieht jedoch nicht nur vor, die Filetstücke des Konzerns Stadt zu verkaufen. Es wird vielmehr auch eine große Zahl städtischer Einrichtungen geschlossen. Dazu zählen insbesondere die städtischen Theater und Museen, das kommunale Kino, die Hälfte der Hallenbäder, die Freizeitheime, die Jugendzentren und das Kongresszentrum. Oper und Kunstmuseum werden nicht geschlossen, sondern vom Land übernommen. Darüber hinaus setzt der Insolvenzverwalter die Einstellung folgender städtischer Aufgaben durch: Beschäftigungsförderung, Wirtschaftsförderung, Wohnungsbauförderung, Kulturförderung.

Alle anderen Aufgaben werden auf ihre zwingenden gesetzlichen Standards zurückgeführt. Die drei bis sechsjährigen Kinder werden zum Beispiel nicht mehr ganztags, sondern nur noch halbtags betreut. Von den 25000 Beschäftigten im Konzern Stadt ist am Ende die Hälfte „privatisiert“; weiteren zehn Prozent wird gekündigt. Die Personalkosten sinken durch diese Maßnahmen um 40 Prozent. Zinsaufwand gibt es keinen mehr, da die Stadt vollständig entschuldet ist. Im Gegenteil, die Stadt kann jetzt Geld anlegen. Mit der Reduzierung der Aufgaben sowie der sozialen Standards sinken zudem die Sach- und Transferausgaben erheblich. Als gegenläufige Effekte bleiben die Aufwendungen für den Sozialplan und der Verlust der Gewinnabführungen insbesondere von den Stadtwerken. 
Als Ergebnis der Insolvenzverwaltung werden die großen Kostenblöcke des städtischen Haushalts deutlich gesenkt: Personalkosten (-30 Prozent), Sachkosten (-10 Prozent), Sozialausgaben (-10 Prozent), Zuwendungen für Vereine und Dritte (-30 Prozent) sowie Zinsen (-100 Prozent); dies entspricht einer Einsparung von insgesamt rund 20 Prozent der städtischen Gesamtausgaben. Ferner werden die Gewerbesteuer um ein Viertel und die Grundsteuer um die Hälfte erhöht. Durch das radikale Konsolidierungsprogramm kann der Haushaltsausgleich selbst dann erreicht werden, wenn die Gewerbesteuer infolge schlechter Konjunktur einbricht. Bei guter Konjunktur und hohen Steuereinnahmen kann die Stadt im erheblichen Umfang Investitionen finanzieren und Rücklagen bilden. Diese Eigenfinanzierung ist allerdings auch notwendig, da die Stadt in absehbarer Zukunft keine Kredite mehr erhält.

Die Insolvenz würde also die grundlegende Sanierung des Haushalts ermöglichen und eine effektive Schuldenbremse bedeuten. Faktisch können die Ausgaben freilich nur mit Hilfe einer Zwangsverwaltung massiv gesenkt werden. Ein Oberbürgermeister würde im Stadtrat nie eine Mehrheit für ein derartig radikales Sparprogramm bekommen. Wie sieht die Stadt ansonsten nach der Insolvenz aus? Es gibt nur noch das Landesmuseum und das Staatstheater, einige private Kunstgalerien und kleinere Privattheater. Die über mehr als 100 Jahre gewachsene Kulturszene mit ihren vielfältigen Einrichtungen und Organisationen implodiert ohne städtische Subvention. Kultur als ein wichtiges Charakteristikum der europäischen Stadt spielt in dieser Kommune nur noch eine untergeordnete Rolle. Der größte Teil der Bevölkerung hat die heimischen Theater und Museen allerdings wohl das letzte Mal in der Schulzeit besucht und wird den Zusammenbruch der Kulturszene vermutlich nicht als großen persönlichen Verlust empfinden.

Unmittelbarer wirken hingegen die Reduzierung der Sportförderung und der Abbau der Standards in der Kinderbetreuung. Eine ganze Reihe von Sportvereinen löst sich auf, weil sie durch fehlende Zuwendungen der Stadt praktisch pleite sind; die verbleibenden Vereine müssen ihre Mitgliedsbeiträge kräftig anheben. Die reduzierte Kinderbetreuung stellt berufstätige Eltern vor fast unlösbare Probleme, die Beschäftigungsquote von Frauen sinkt daher deutlich. Generell steigt die Arbeitslosigkeit kräftig an, da die meisten vormals bei der Stadt beschäftigten Mitarbeiter Probleme haben, einen neuen Arbeitsplatz zu finden. Auch die neuen Eigentümer der vormaligen Kommunalunternehmen bauen massiv Personal ab. Beim Erwerb der Stadtwerke ging es ihnen vor allem um den lokalen Absatzmarkt. Der Flughafen ist nur noch die Dependance eines großen internationalen Flug- 
hafens. Das gleiche gilt für die Messe. Die privaten Investoren verlieren dort schnell die Geduld und verkaufen sie an eine andere Messegesellschaft.

Einen großen Teil der durch den Sparkurs der Stadt verursachten Arbeitslosigkeit hat die Bundesagentur für Arbeit zu bewältigen. Auf die Kommune kommen dagegen die erhöhten Kosten der Unterkunft für die Hartz IVEmpfänger zu. Zudem wird erst jetzt deutlich, wie viele hauptamtliche Stellen bei den Wohlfahrtsverbänden offenbar von der Stadt bezahlt worden sind. Denn auch diese müssen nach der Kürzung der städtischen Zuschüsse massiv Personal abbauen. Last but not least, kümmert sich nur noch die Polizei um sozial auffällige Personen. Die Stadt hat sich aus der aktiven Sozialarbeit weitgehend zurückgezogen. Streetworker und aufsuchende Sozialarbeiter gibt es keine mehr. Jugendzentren sind dichtgemacht. Soziale und kulturelle Verarmung sind der Preis für die Rosskur bei den städtischen Finanzen.

Rechtlich dürfte die Kommunal- und Länderinsolvenz relativ leicht umzusetzen sein. Möglicherweise müsste nur ein Satz im Insolvenzrecht gestrichen werden ${ }^{20}$. Natürlich ginge es bei einer Kommunalinsolvenz darum, die Stadt zu sanieren und nicht aufzulösen, denn zumindest die Pflichtaufgaben sind weiter zu erfüllen. Anstatt einer Auflösung käme allenfalls die Zusammenlegung mit einer anderen Kommune in Betracht. Wenn als Folge der Insolvenzfähigkeit Landkreise oder Bundesländer fusionieren müssten, wäre dies sicherlich ein gutes Ergebnis; denn von beiden Gebietskörperschaften gibt es zu viele.

Der Sanierungsansatz ist eine Möglichkeit, um von einem zu hohen Ausgabenniveau herunterzukommen und die Steuersätze deutlich anzuheben. Im normalen Politik- und Verwaltungsgeschäft sind harte Einschnitte und kräftige Steuererhöhungen kaum durchzusetzen. Schließungen von Museen oder betriebsbedingte Kündigungen sind Tabus. Vermutlich würde es aber gar nicht oft zu einer Insolvenz kommen, da allein die Möglichkeit einer solchen eine disziplinierende Wirkung entfalten dürfte. Durch die rechtliche Unmöglichkeit einer Insolvenz läuft im Grunde alles so weiter wie bisher, auch wenn die Kredite exponentiell steigen. Um die Defizitentwicklung zu begrenzen, kann der Finanzverantwortliche nur auf seine Überzeugungskraft setzen oder mit Rücktritt drohen. Die sozialen und kulturellen Folgen einer Insolvenz könnten hingegen eine Drohkulisse bilden, die keinen politischen Akteur kalt ließe. Die Haushaltszahlen wären dann nicht mehr nur das Problem des Finanzministers oder Kämmerers, sondern würden auch in der Fachpolitik auf mehr Interesse stoßen.

\footnotetext{
${ }^{20}$ Vgl. Blankart, Föderalismus, S. 208f.
} 
Der Vergleich zur freien Wirtschaft soll diesen Punkt verdeutlichen. In einem Unternehmen, das trotz hoher und vor allem dauerhafter Verluste einen Anteilseigner hat, der Kapital immer klaglos und in unbegrenzter Höhe nachschießt, würden vermutlich weder eine strategische Neuausrichtung noch harte Kostensenkungsprogramme umgesetzt werden. Auch in einem Unternehmen sind Führungskräfte und Beschäftigte keineswegs automatisch betriebswirtschaftlich denkende Menschen. Ein deutscher Ingenieur besitzt vor allem den Ehrgeiz, gute Maschinen zu entwickeln, während der Preis und die Bedürfnisse des Nutzers ihn weniger interessieren. Da unterscheidet er sich nicht von den Sachbearbeitern in der Fachverwaltung, die immer neue Programme entwickeln und ihr Budget ständig erweitern möchten. Nur der zu erwirtschaftende Deckungsbeitrag unterscheidet das Unternehmen von der öffentlichen Verwaltung. Wird dieser nicht erzielt, steht zunächst das flexible Gehalt und im zweiten Schritt auch der Arbeitsplatz zur Disposition. Wenn es ernst wird, finden Menschen erfahrungsgemäß Lösungen, die vorher kaum möglich schienen. So wäre es möglicherweise auch, wenn einer Gebietskörperschaft die Insolvenz drohte. Darüber hinaus bestünde ein teils kritischer, teils positiver Effekt darin, dass die öffentliche Hand nicht mehr unbeschränkt Kredite erhielte oder sich Kredite zumindest im Einzelfall deutlich verteuerten. Die Banken würden genauer prüfen, ob beispielsweise eine Kommune noch kreditwürdig ist und in welcher Höhe ein Risikoaufschlag fällig wäre. Letzteres würde die Haushaltssituation erst einmal verschlechtern, da es momentan keine günstigere Finanzierung als einen deutschen Kommunalkredit gibt. Das würde sich bei einer Insolvenzmöglichkeit ändern. Möglicherweise würden die politischen Entscheidungsträger dann auf nicht unbedingt nötige Ausgaben verzichten oder eher Steuern erhöhen, als teure Kredite aufzunehmen. Die politische Rationalität könnte sich aufgrund der Insolvenzmöglichkeit der betriebswirtschaftlichen Rationalität beziehungsweise dem gesunden Menschenverstand annähern.

\section{Inflation}

Währungen werden zum Zweck der Staatsfinanzierung manipuliert, seitdem es Geld gibt. So reduzierten beispielsweise die Römer den Kupfergehalt ihrer Münzen während der Punischen Kriege erheblich ${ }^{21}$. Dadurch bezahl-

\footnotetext{
${ }^{21}$ Vgl. hierzu und zu weiteren Inflationen in der Geschichte Peter Bernholz, Monetary Regimes and Inflation. History, Economic and Political Relationships, Cheltenham/ Northampton 2003.
} 
ten sie einen Großteil ihrer Schulden nicht, was faktisch einem Staatsbankrott gleichkam. Auch im 20. Jahrhundert setzten Staaten häufig auf Inflation, um Kriegskosten zu finanzieren. Das wohl berühmteste Beispiel ist Deutschland zwischen 1914 und 1923. Obwohl Reichsfinanzminister Matthias Erzberger 1920 den Staatsbankrott verhindern wollte, kam ihm die Inflation entgegen, um die Basiskompromisse der Weimarer Republik finanzieren zu können ${ }^{22}$. Die Demobilisierung, der Ausbau des Sozialstaats sowie der Schuldendienst für die Kriegsanleihen erforderten einen ungeheuren Finanzbedarf, der durch Steuern und fundierte Kreditaufnahme kaum zu decken war.

Wenn der Staat eine relativ hohe Inflation akzeptiert beziehungsweise sogar verursacht, besteht die Gefahr, dass die Situation nicht mehr beherrschbar wird und die Währung ihre Funktion verliert. Genau das war im Deutschen Reich spätestens ab 1922 der Fall. Die Lage geriet außer Kontrolle, die Inflation wurde zur Hyperinflation, die Währung wurde durch eine neue ersetzt. Der Staat war seine Schulden erst einmal los. Der Preis dafür war aber hoch. Ein großer Teil der Mittelschicht war verarmt und hatte nur wenig Vertrauen in die junge Demokratie. Die infolge der Inflation schwache Ausstattung der Banken und Unternehmen mit Eigenkapital wirkte sich in der Wirtschaftskrise besonders verhängnisvoll aus. Aufgrund der Verwüstung des Kapitalmarkts war das Zinsniveau hoch ${ }^{23}$, was zusammen mit der „Angst vor der Inflationsangst ${ }^{\text {“24 }}$ den Handlungsspielraum der Politik ab 1929 stark einschränkte.

Die Finanzierung der amerikanischen Kriege seit dem 11.September 2001, die Flutung der Geldmärkte mit Liquidität sowie die überraschende Rückkehr der Konjunkturpolitik als Konsequenz der Wirtschafts- und Finanzkrise von 2008/09 lösen erneute Inflationsängste aus. Absolut besorgniserregend ist dabei vor allem, dass die Notenbanken in großem Stil Staatsanleihen aufkaufen. Sollten die Regierungen diesen Weg weiter gehen wollen und eine relativ hohe Inflation akzeptieren oder sogar forcieren, um ihre Schulden zu reduzieren, wäre das, wie die Geschichte lehrt, ein Spiel mit dem Feuer. Am Ende könnten der Staatsbankrott und eine neue Währung stehen.

${ }^{22}$ Vgl. Detlev J. K. Peukert, Die Weimarer Republik. Krisenjahre der klassischen Moderne, Frankfurt a. M. 1987, S. 72 f.

${ }^{23}$ Vgl. Theodore Balderston, Links between Inflation and Depression: German Capital and Labour Markets, 1924-1931, in: Gerald D. Feldman (Hrsg.), Die Nachwirkungen der Inflation auf die deutsche Geschichte 1924-1933, München 1985, S. 157-185.

${ }^{24} \mathrm{Vgl}$. Knut Borchardt, Das Gewicht der Inflationsangst in den wirtschaftspolitischen Entscheidungsprozessen während der Weltwirtschaftskrise, in: Feldman (Hrsg.), Nachwirkungen der Inflation, S. 233-260. 


\section{Nachhaltige Finanzpolitik}

Wenn die Ziele der Schuldenbremse ohne Inflation erfüllt werden sollen, bleibt als Lösungsansatz nur eine nachhaltige Finanzpolitik. Dieses Prinzip bedeutet, nicht mehr Ressourcen zu verbrauchen, als zur Verfügung stehen, und gilt ausdrücklich auch für die langfristigen Renten- und Pensionsverpflichtungen. Um ohne Nettoneuverschuldung auszukommen, gibt es nur ein Patentrezept: Ausgaben reduzieren und Einnahmen erhöhen. Am besten würde der Staat sogar auf eine Neuverschuldung in Höhe der Tilgung verzichten und sich somit im Laufe einer Generation entschulden; das wäre allerdings ein äußerst ehrgeiziges Ziel. Bereits bei einem strukturell ausgeglichenen Haushalt, also bei einer nicht mehr steigenden Verschuldung, würde der Staat aus seinen Schulden herauswachsen, wie dies zum Beispiel Großbritannien im 19. Jahrhundert gelang, den USA nach dem Zweiten Weltkrieg oder Belgien nach $1995^{25}$.

Für die Politik ist das „Herauswachsen“ aus den Schulden ein verführerisch einfaches Konzept. Ab einem gewissen Ausgabenniveau reicht es in der Regel jedoch nicht, allein auf Wirtschaftswachstum zu setzen. Die in einer Phase des konjunkturellen Aufschwungs sprudelnden Steuereinnahmen decken häufig gerade einmal die Ausgaben wie 2007/08 in Deutschland, obwohl sie eigentlich hohe Überschüsse verursachen müssten. Entwickelt sich die Konjunktur weniger gut, entstehen automatisch hohe Defizite. Um den Haushalt auch bei einer mittleren Konjunkturentwicklung auszugleichen, muss entweder das Ausgabeniveau reduziert oder die Steuerlast erhöht werden.

Auf der Ausgabenseite dürften sich freilich auch in Zukunft kaum echte Kürzungen durchsetzen lassen. Allerdings wären eine Neuausrichtung der Sozialpolitik nach dem Grundsatz Vor- statt Nachsorge sowie die Begrenzung des Anstiegs der Transferausgaben richtig. Das Rentenniveau muss wahrscheinlich noch weiter nach unten korrigiert werden. Das würde das teuerste Wahlgeschenk der bundesdeutschen Geschichte - Adenauers dynamische Rente - endgültig revidieren und die Generationenbilanz erheblich verbessern. Hingegen dürfte der Umstieg auf ein kapitalgedecktes Verfahren zu lange dauern, um in absehbarer Zukunft eine fiskalische Entlastung zu bewirken. Das gilt im Übrigen auch für andere öffentliche Ausgaben. Es wird noch Jahrzehnte dauern, bis die seit einigen Jahren geförderten Stiftungen öffentliche Ausgaben übernehmen können. Im Deutschen Kaiserreich gab

\footnotetext{
${ }^{25}$ Vgl. Wagschal/Wenzelburger, Haushaltskonsolidierung, S. 67-79.
} 
es bereits eine Stiftungskultur, die jedoch unter den beiden Inflationen und später unter der zumindest gefühlt hohen Besteuerung stark gelitten hat. Demgegenüber konnten in den USA ungestört von Hyperinflationen und Staatsbankrotten Universitäten wie Harvard oder Princeton mittels Spenden einen Kapitalstock aufbauen, dessen Verzinsung - zumindest wenn das Geld nicht spekulativen Anlagen zum Opfer gefallen ist - einen nicht unerheblichen Teil des jährlichen Budgets trägt.

Bei den Ausgaben geht es nicht in erster Linie um Kürzungen, sondern um die Reduzierung der Steigerungsraten und um Umschichtungen in Richtung eines „vorsorgenden Sozialstaats“. Neben der Rente sei als weiteres Beispiel das Kindergeld genannt, das langfristig eingefroren werden könnte. Stattdessen sollte das Geld für den Ausbau der Kinderbetreuung ausgegeben werden. Denn nicht nur die stetig wachsende Quantität der Ausgaben ist problematisch, sondern auch ihre sinkende Qualität. So steigt der Anteil der Zinsen und der Transferausgaben, insbesondere für die Rente, während der Anteil für Bildung, Wissenschaft und Investitionen sinkt. Im Jahr 2007 waren bereits zwei Drittel des Bundeshaushalts durch Transferausgaben und Zinsen festgelegt.

Fakt ist, dass ein skandinavisches Ausgabenniveau und eine angelsächsische Steuerquote nicht gleichzeitig zu haben sind. Wenn Kürzungen bei den Sozialausgaben nicht durchsetzbar sind, müssen die Abgaben erhöht werden. Da sich die Sozialabgaben bereits auf einem hohen Niveau befinden, kommt nur die Erhöhung der Steuerquote in Frage. Diese stagniert in Deutschland seit über 70 Jahren und ist auch im internationalen Vergleich nicht besonders hoch. Bevor die Tarife erhöht werden, sind zunächst die Bemessungsgrundlagen der großen Steuern grundlegend zu überarbeiten. Ausnahmetatbestände und Steuersubventionen sollten dabei weitgehend abgeschafft werden. Damit der Gewinn nicht mehr klein gerechnet werden kann, ist die Besteuerung der Unternehmen ertragsunabhängiger zu gestalten. Möglicherweise würde bei einer soliden Bemessungsgrundlage bereits die Erhöhung der Steuerquote um ein oder zwei Prozentpunkte ausreichen, um den Haushalt strukturell auszugleichen. Infrage käme ein Mix aus einer Erhöhung der Mehrwertsteuer, der Einkommen- und vor allem der Unternehmensteuern.

Schweden und Finnland sind Beispiele dafür, wie eine nachhaltige Finanzpolitik erfolgreich sein kann, ohne dass der soziale Anspruch aufgegeben werden muss. In der ersten Hälfte der 1990er Jahre hatten schwedische Produkte große Schwierigkeiten, sich auf dem Weltmarkt zu behaupten. Nahezu alles - Industrieunternehmen, Banken und Währung sowie der 
Wohlfahrtsstaat - geriet damals in die Krise. Vor dem Hintergrund dieser fundamentalen Krise ist eine bemerkenswerte Kehrtwende gelungen ${ }^{26}$. Strukturreformen, moderate Ausgabenkürzungen, Einnahmenerhöhungen und eine gute Konjunktur haben schließlich zu massiven und dauerhaften Haushaltsüberschüssen geführt. Dabei ist der Staat keinesfalls kaputt gespart worden. Schweden ist kein Niedrigsteuerland. Es finanziert seine im internationalen Vergleich nach wie vor hohen Ausgaben durch eine entsprechend hohe Abgabenquote. Eine ähnliche Entwicklung hat auch sein Nachbar Finnland erlebt. Von den ökonomischen Verwerfungen nach dem Ende des Kalten Kriegs hart getroffen, hat Finnland seine Strukturen grundlegend geändert und damit ebenfalls eine Phase der Haushaltsüberschüsse eingeleitet. Das Land ist sogar zum viel gerühmten PISA-Sieger aufgestiegen, nicht zuletzt weil es von seinen sozialen Transferausgaben in Richtung Bildung umgeschichtet hat.

Zur nachhaltigen Finanzpolitik gehört auch eine Reform des Fiskalföderalismus. Insbesondere die Steuerautonomie der Länder ist unbedingt zu stärken. Das wäre die logische Konsequenz der Schuldenbremse, die den Ländern mit der Kreditaufnahme ihre einzige flexible Einnahmequelle genommen hat ${ }^{27}$. So könnte den Ländern bei der Einkommensteuer die Möglichkeit eingeräumt werden, Zuschläge zu erheben ${ }^{28}$. Die Mehrwertsteuer würde dann zur reinen Bundessteuer. Durch dieses steuerliche Trennsystem und eine Reduzierung der Ausgleichsquote könnten die Fehlanreize im Länderfinanzausgleich deutlich abgebaut werden. Zudem entstünden leistungsfähigere Strukturen, wenn die Anzahl der Bundesländer halbiert würde. Die strukturelle Krise der Kommunalfinanzen könnte weitgehend beseitigt werden, indem die Gewerbesteuer ausgebaut, die Grundsteuer auf eine aktuelle Bemessungsgrundlage gestellt und ein Teil der Sozialausgaben vom Bund

${ }^{26}$ Vgl. ebenda, S. 79-88. Überaus interessant ist der Erfahrungsbericht des ehemaligen schwedischen Finanzministers Jens Henriksson, Ten Lessons about Budget Consolidation, Brüssel 2007.

${ }^{27}$ Vgl. Wolfgang Renzsch, Die zweite Stufe der Bundesstaatsreform, in: Egle/Zohlnhöfer (Hrsg.), Zweite Große Koalition, S. 455-462, hier S. 459f.

${ }^{28}$ Viele Politiker betrachten Einkommensteuerzuschläge mit Skepsis, da sie Steuerdumping und eine Abkehr vom Leitbild der Gleichwertigkeit der Lebensverhältnisse befürchten. Clemens Fuest (Steuerwettbewerb unter den Bundesländern - wären die finanzschwachen Länder die Verlierer?, in: Konrad/Jochimsen (Hrsg.), Föderalismuskommission II, S. 119-133, hier S. 132) gelangt jedoch zu dem Schluss, „dass gängige ökonomische Argumente eher dagegen als dafür sprechen, dass die Einführung eines variablen Zuschlags der Länder zur Einkommensteuer das wirtschaftliche Gefälle zwischen ,reichen' und ,armen' Bundesländern verstärken". 
übernommen würde ${ }^{29}$. Beim letzten Punkt ist mit der Übernahme der Grundsicherung im Alter und bei Erwerbsminderung durch den Bund bereits 2011 eine zentrale Maßnahme eingeleitet worden.

${ }^{29}$ Vgl. Hansmann, Einnahmenproblem, S. $241 \mathrm{f}$. 